\title{
Medikation statt Fixierung - noch ist das illegal
}

\author{
Seit Juli dürfen Psychiater Patienten zwar gegen ihren Willen verwah- \\ ren und notfalls auch fixieren, aber nicht mehr medikamentös behan- \\ deln. Eine gesetzliche Neuregelung soll nun die Zwangsbehandlung \\ psychisch Kranker als Ultima Ratio wieder legalisieren.
}

\begin{abstract}
eit gut einem halben Jahr liegt den Psychiatern ein Beschluss des Bundesgerichtshofs (BGH) schwer im Magen: Psychisch Kranke dürfen nicht mehr gegen ihren Willen medizinisch behandelt werden, weil dafür nach zwei vorausgegangenen Urteilen des Bundesverfassungsgerichts (BVG) die Rechtsgrundlage fehlt. Was in vielen Medien als Erfolg für die Autonomie der Patienten gefeiert wurde, hat aber eine gravierende Schattenseite: Patienten, deren Autonomie und Einwilligungsfähigkeit durch eine psychische Erkrankung stark beeinträchtigt ist, werden nun teilweise gar nicht mehr behandelt.
\end{abstract}

\section{Dauerhafte Heimeinweisung?}

Welche Auswirkungen das haben kann, hat Professor Tilman Steinert vom Zentrum für Psychiatrie Südwürttemberg am Beispiel einer Patientin mit bipolarer Störung erläutert. Sie wurde in einer schweren Krankheitsphase bislang anfangs auch gegen ihren Willen behandelt, worauf sich der Zustand besserte und sie anschließend die Therapie akzeptierte. In der Regel konnte sie nach vier Wochen entlassen werden. Nach den Urteilen des BVG und BGH ist eine initiale Therapie gegen den Willen der Patientin nun aber nicht mehr möglich. Die Behandlung, die ihre Einwilligungsfähigkeit wieder herstellen sollte, muss ihr verwehrt bleiben. Die Konsequenz: Die Frau wird nun schon mehrere Monate unbehandelt in der Klinik verwahrt. „Ich frage mich, wie lange das die Kassen noch finanzieren, wenn keine Behandlung stattfindet", sagte Steinert. „Es besteht nun die Gefahr, dass die Patientin in einem geschlossenen Heim untergebracht werden muss. Das wäre natürlich eine Katastrophe.“
Noch drastischer ist die Situation bei Anorexiepatienten. „Nach gegenwärtiger Rechtslage ist eine Zwangsernährung nur möglich, wenn die Patienten bereits das Bewusstsein verloren haben. Dann misslingt eine Lebensrettung jedoch meistens“, stellt die DGPPN in einem Memorandum fest. Lassen Ärzte die Patienten jedoch sterben, droht ihnen eine Klage wegen unterlassener Hilfeleistung.

Inzwischen haben Psychiater diesen Missstand immer wieder offen kritisiert und den Gesetzgeber dazu aufgefordert, eine klare rechtliche Grundlage zu schaffen. Dabei begrüßen sie zunächst das BGH-Urteil in dem Sinne, dass einwilligungsfähige Patienten, etwa im Maßregelvollzug, nicht gegen ihren Willen behandelt werden dürfen - solche Fälle waren Auslöser für die aktuellen Urteile. Psychiater um Dr. Sabine Müller von der Charité in Berlin halten es aber geradezu für unterlassene Hilfeleistung und eine Missachtung der Autonomie der Patienten, wenn Ärzte „bei einer tiefgreifenden Störung der Willensbildung Maßnahmen zur Heilung und zur Wiederherstellung der Autonomiefähigkeit unterlassen“. Sie kritisieren zudem, dass die aktuellen Gerichtsurteile Menschen mit psychischen Erkrankungen eine Selbstverantwortung abverlangen, zu der diese häufig nicht in der Lage sind [Der Nervenarzt 2012; 9:1150-55]. Dem schließt sich auch Steinert an: „Wir wollen nicht, dass Menschen abgeschoben und in Spezialeinrichtungen verwahrt werden, weil sie krankheitsbedingt die Notwendigkeit einer Behandlung nicht einsehen können. Wir wollen auch nicht, dass mechanischer Zwang wie Fixieren oder Einsperren an die Stelle einer Behandlung tritt und die Pati- enten damit noch stärker als bei einer Medikation traumatisiert werden. Wir wollen nicht, dass unbehandelte Menschen mit schweren psychischen Erkrankungen sich selbst töten oder Straftaten begehen und dann mit einer potenziell unbefristeten Unterbringung in der forensischen Psychiatrie einen viel größeren Eingriff in ihre Freiheitsrechte hinnehmen müssen, als bei einer kurzen, initial gegen ihren Willen stattfindenden Behandlung. “

\section{Gutachter soll zustimmen}

Immerhin scheint die Bundesregierung das Problem erkannt zu haben. Am 19. November, kurz vor Beginn des DGPPNKongresses, hat die Koalition einen Gesetzentwurf vorgelegt, der die Gemüter der Psychiater etwas beruhigen dürfte. Beim geplanten „Gesetz zur Regelung der betreuungsrechtlichen Einwilligung in eine ärztliche Zwangsmaßnahme" wurden viele Eingaben der DGPPN so aufgenommen, „dass das Gesetz in der Praxis auch umsetzbar scheint", sagte DGPPN-Präsident Peter Falkai auf dem Kongress. Kritik äußerte die Gesellschaft jedoch am geplanten externen Gutachter, der nach dem Entwurf in eine Zwangsbehandlung einwilligen muss. „In der Praxis könnte das sehr schwierig werden, vor allem in ländlichen Gebieten, wo es nicht sehr viele Psychiater gibt. Wenn es wochenlang dauert, bis ein Gutachter gefunden wird, und die Patienten unbehandelt eingesperrt werden, dann ist das keine gute Lösung “, sagte Steinert.

Selbstkritik war allerdings auch zu hören. So hätten es die Psychiater versäumt, eigene ethische Prinzipien zu entwickeln, wie sich Ärzte in Konfliktsituationen verhalten sollten, wenn sie die Autonomie des Patienten respektieren und gleichzeitig seinem Wohlergehen dienen wollen, sagte der „President Elect“ der DGPPN, Professor Wolfgang Maier. Inzwischen wurde eine Taskforce eingesetzt, um hierfür ethische Grundsätze zu erarbeiten. „Wir wollen künftig nicht einfach defensiv auf Gesetzgebungsvorhaben und Gerichtsbeschlüsse reagieren, sondern auch proaktiv in einer solchen Diskussion mitwirken“, so Maier.

Thomas Müller

Pressekonferenz "Selbstbestimmung und Patientenautonomie in der Psychiatrie."

DGPPN-Kongress, Berlin, 23.11.2012 